\title{
Traumas múltiplos em vítimas de traumatismo craniencefálico
}

\author{
José Roberto Tude Melo*, Ricardo Araújo da Silva**, Tiago Ribeiro***, \\ Edson Duarte Moreira Júnior ${ }^{\star \star \star \star}$ \\ Hospital Geral do Estado da Bahia e Curso de Pós-Graduação em Medicina e Saúde da Universidade Federal da Bahia (UFBA)
}

\section{RESUMO}

Objetivos: verificar a freqüência de pacientes com trauma craniencefálico que apresentaram lesões em outros locais do corpo, assim como definir quais os segmentos mais afetados e principais causas de trauma neste grupo. Metodologia: estudo descritivo, tipo corte transversal, através da revisão de 555 prontuários médicos de vitimas de trauma craniano internadas no Hospital Geral do Estado da Bahia, durante o ano de 2001. Trabalho avaliado e aprovado pela diretoria do hospital, assim como pelo Comitê de Ética em Pesquisa. Conclusão: os traumas múltiplos foram observados em 31,9\% das vitimas de trauma craniano e tiveram, como principal causa, o atropelamento, sendo as lesōes faciais e ortopédicas dos membros as mais freqüentes.

\section{PALAVRAS-CHAVE}

Epidemiologia. Trauma craniencefálico. Trauma múltiplo.

\section{ABSTRACT \\ Multiple traumas in head injured patients observed in association with head injury. \\ KEYWORDS \\ Epidemiology. Craniocerebral trauma. Multiple trauma.}

Objective: to identify the association between head trauma and other injuries of the body, define the most frequent segment of the body affected in this association and the major cause of trauma in this group. Methodology: assessment and notification of 555 medical files of head trauma cases and identify those who had multiple injuries, admitted at the General Hospital of Bahia, Brazil, during the year of 2001. Conclusion: multiple traumas were observed in $31.9 \%$ of head injured patients, usually with pedestrian traffic accidents. Facial and limb orthopedic trauma were the most frequent injuries

\section{Introdução}

Em torno de $37 \%$ dos pacientes admitidos em Unidades de Emergência são vítimas de trauma mecânico. O sucesso no atendimento e evolução desses doentes difere de acordo com o tipo de trauma e a população atingida. As principais causas de traumatismo craniencefálico (TCE) são os acidentes envolvendo os meios de transporte, destacando-se automóveis, motocicletas, bicicletas e incluindo os atropelamentos. Esses dados podem diferir um pouco, dependendo da região e da população estudadas ${ }^{10,11}$.

Com o crescimento das metrópoles, observa-se aumento na incidência dos traumas secundários à agressão física, decorrentes da violência urbana ${ }^{10}$, que participam com maiores proporções nas classes sociais menos favorecidas. Em sentido amplo, a agressão física é resultado de conduta caracterizada pelo intuito destrutivo, de ofensa, ataque e hostilidade. A agressão física foi observada como principal causa de TCE leve

\footnotetext{
*Neurocirurgião do Hospital São Rafacl e do Hospital Geral do Estado da Bahia. Mestrando do Curso de Pós-Graduação da Faculdade de Medicina da UFBA.

${ }^{* *}$ Mestre em Deontologia e Odontologia Legal pela Universidade de São Paulo.

***A Acadêmico da Faculdade de Medicina da UFBA.

****Diretor em excrcicio da Diretoria Cientifica do Hospital São Rafael. Pesquisador associado da FIOCRUZ.
} 
na cidade de Curitiba, no Paraná, precedendo às quedas de altura, aos acidentes automobilísticos, às quedas da própria altura e ao atropelamento ${ }^{3}$.

As lesões intracranianas secundárias aos ferimentos por arma de fogo exibem algumas peculiaridades, como maiores taxas de morbidade e letalidade, que podem atingir valores acima de $90 \%$ quando a vítima apresentar pontuação na escala de coma de Glasgow abaixo de 5. Esse tipo de lesão predomina em adultos jovens do sexo masculino ${ }^{15}$.

Outras causas importantes de TCE são as quedas, ocorrendo principalmente entre crianças e idosos ${ }^{10}$. No Reino Unido, cerca de $10 \%$ dos atendimentos em Unidades de Emergência são constituídos de casos de trauma craniano, e as quedas correspondem a $40 \%$ destas admissões ${ }^{8}$.

São citadas como principais causas de TCE os acidentes com meios de transporte, as agressões físicas, as quedas e as lesões por arma de fogo, além de outras menos freqüentes ${ }^{2,3,8,10,11,13}$.

Buduhan e McRitchie ${ }^{5}$, em 2000, avaliaram 645 pacientes politraumatizados e constataram que 46 vítimas apresentavam lesões não detectadas (traumas múltiplos), com um total de 63 injúrias. A maioria foi atendida com pontuação menor ou igual a 8 na escala de coma de Glasgow, e as lesões menos diagnosticadas foram aquelas dos membros (sistema musculoesquelético) e cabeça. Melo e cols. ${ }^{13}$, em 2003, constataram, em 431 vítimas estudadas, que $220(51 \%)$ apresentavam traumas associados ao trauma do crânio, sendo os traumas ortopédicos dos membros os mais freqüentes, seguidos dos da face. Essa associação também foi evidenciada por Sala e cols. ${ }^{14}$, em 2000, principalmente em vítimas de acidentes com meios de transporte.

O escopo deste trabalho foi $o$ de definir a freqüência das associações entre traumas cranianos e os de outras regiões do corpo, quais as principais causas das lesões múltiplas, assim como verificar os principais sítios de lesão e relação quanto à gravidade do trauma craniano.

\section{Casuística e método}

Trata-se de estudo descritivo, do tipo corte transversal, por meio da revisão de 555 prontuários médicos de vítimas de TCE internadas no Hospital Geral do Estado da Bahia (HGE) entre janeiro e dezembro de 2001.

A escolha do HGE deve-se ao fato de ser o hospital de referência no estado da Bahia para o atendimento de politraumatizados (dados da Secretaria de Saúde do Estado da Bahia). Foram incluídas, na pesquisa, todas as vítimas de TCE cujo registro constasse no Livro de Prontuários de 2001, documento disponível no Serviço de Arquivo Médico do HGE.

Os dados obtidos da revisão dos prontuários foram transferidos para questionário composto dos seguintes itens: registro, idade, sexo, data da ocorrência, localidade (capital ou interior), causa do trauma, achado radiológico, associação com outros traumas, tratamento estabelecido, infecção durante o período de internação, associação com bebida alcoólica, óbito, alta e a pontuação na escala de coma de Glasgow na admissão e na alta hospitalar.

O critério de inclusão na amostra do estudo foi a anotação, em prontuário, do diagnóstico de TCE associado a trauma em outra região do corpo, comprovado por exame médico do especialista envolvido no atendimento da vítima ou pelos métodos diagnósticos por imagem (radiografia simples, tomografia computadorizada ou ultra-sonografia).

Os dados foram compilados em software preestabelecido (SPSS - Statistical Software Program versão 9.0) com o objetivo principal de se obter análise panorâmica da distribuição atual das associações dos traumas craniencefálicos com os de outras regiões anatômicas, suas vítimas e características na população estudada.

A pesquisa foi aprovada pela diretoria do hospital, assim como pelo Comitê de Ética em Pesquisa da Universidade Federal da Bahia, conforme parecer final $n^{\circ} 17 / 2003$.

\section{Resultados}

Verificou-se que em 177 (31,9\%) dos 555 prontuários havia descrição de trauma em outra região do corpo (lesões concomitantes ao trauma craniano); as principais associações observadas foram os traumas na face $(30,5 \%)$, seguida das lesões ortopédicas nos membros (22\%), lesões de tórax $(6,2 \%)$, da coluna cervical $(4 \%)$ e trauma abdominal $(3,4 \%)$ (Tabela 1$)$.

Entre as vítimas de traumas múltiplos, a maioria era do sexo masculino $(80,2 \%)$, com idade compreendida entre 21 e 30 anos, seguida daqueles entre 11 e 20 anos de idade $(23,7 \%)$. As principais causas de lesões em múltiplos segmentos foram os acidentes com meios de transporte $(53,1 \%)$, seguidos das agressões físicas com ou sem armas $(19,2 \%)$ e quedas $(16,4 \%)$ (Tabela 2). Quanto à complexidade das associações, foi verificado que $117(66,1 \%)$ pacientes apresentavam apenas uma lesão em outro local além do crânio, 40 $(22,6 \%)$ sofreram trauma em mais dois locais, e em 20 $(11,3 \%)$ traumatizados observou-se mais de três sítios associados de lesão concomitantes ao TCE (Tabela 3). 
Tabela 1

Principais lesões concomitantes ao trauma craniano

\begin{tabular}{lcc}
\hline Local & $\mathbf{n}$ & $\%$ \\
\hline Face & 54 & 30,5 \\
Ortopédico* & 39 & 22 \\
Tórax & 11 & 6,2 \\
Coluna cervical & 7 & 4 \\
Abdome & 6 & 3,4 \\
Outros & 60 & 33,9 \\
Total & $\mathbf{1 7 7}$ & $\mathbf{1 0 0}$ \\
\hline
\end{tabular}

* Lesỏes ósseas em membros superiores e/ou inferiores.

Dados colhidos em prontuários médicos do Hospital Geral do Estado da Bahia.
Tabela 3

Distribuição dos casos em função do número de segmentos corpóreos com lesões, além do crânio

\begin{tabular}{lcc}
\hline $\begin{array}{l}\text { Lesões } \\
\text { associadas }\end{array}$ & Casos & Morbidade \\
\hline 1 & $117(66,2 \%)$ & $27(23,1 \%)$ \\
2 & $40(22,6 \%)$ & $4(10 \%)$ \\
$>3$ & $20(11,3 \%)$ & $3(15 \%)$ \\
Total & $177(100 \%)$ & $\mathbf{3 4}(\mathbf{1 9 , 2} \%)$ \\
\hline
\end{tabular}

Tabela 2

Características de 177 pacientes vítimas de traumas múltiplos em Salvador, BA, 2001

\begin{tabular}{|c|c|c|c|c|c|c|}
\hline \multirow[t]{2}{*}{ Características das vítimas } & \multicolumn{2}{|c|}{ Homens } & \multicolumn{2}{|c|}{ Mulheres } & \multicolumn{2}{|c|}{ Total } \\
\hline & $\mathbf{n}$ & $\%$ & n & $\%$ & $\mathrm{n}$ & $\%$ \\
\hline \multicolumn{7}{|l|}{ Faixa etária (em anos) } \\
\hline$\leq 10$ & 10 & 47,6 & 11 & 52,4 & 21 & 11,9 \\
\hline 11 a 20 & 35 & 83,3 & 7 & 16,7 & 42 & 23,7 \\
\hline 21 a 30 & 42 & 91,3 & 4 & 8,7 & 46 & 26,0 \\
\hline 31 a 40 & 28 & 82,4 & 6 & 17,6 & 34 & 19,2 \\
\hline 41 a 50 & 14 & 73,7 & 5 & 26,3 & 19 & 10,7 \\
\hline 51 a 60 & 9 & 81,8 & 2 & 18,2 & 11 & 6,2 \\
\hline$\geq 61$ & 4 & 100 & 0 & 0 & 4 & 2,3 \\
\hline Total & 142 & 80,2 & 35 & 19,8 & 177 & 100 \\
\hline \multicolumn{7}{|l|}{ Causas do trauma } \\
\hline Atropelamento & 35 & 79,5 & 9 & 20,5 & 44 & 24,9 \\
\hline Acidente automobilístico & 21 & 75,0 & 7 & 25,0 & 28 & 15,8 \\
\hline Queda de altura & 20 & 74,1 & 7 & 25,9 & 27 & 15,3 \\
\hline Acidente motociclistico & 17 & 77,3 & 5 & 22,7 & 22 & 12,4 \\
\hline Ferimento por arma de fogo & 14 & 77,8 & 4 & 22,2 & 18 & 10,2 \\
\hline Agressão física & 15 & 93,8 & 1 & 6,3 & 16 & 9,0 \\
\hline Outros & 10 & 90,9 & 1 & 9,1 & 11 & 6,2 \\
\hline Queda da própria altura & 2 & 100 & 0 & 0 & 2 & 1,1 \\
\hline Desconhecida & 8 & 5,6 & 1 & 2,8 & 9 & 5,1 \\
\hline Total & 142 & 100 & 35 & 100 & 177 & 100 \\
\hline
\end{tabular}

Dados colhidos em prontuários médicos do Hospital Geral do Estado da Bahia.

No momento da admissão na sala de emergência, $46,6 \%$ das vítimas de traumas múltiplos foram atendidas com diagnóstico de TCE grave (pontuação menor ou igual a 8 na escala de coma de Glasgow), $33,6 \%$ com TCE leve (Glasgow entre 14 e 15) e 19,8\% com TCE moderado (Glasgow entre 9 e 13).

\section{Discussão}

O predomínio de adultos jovens do sexo masculino entre as vítimas de traumas mecânicos, observado no presente estudo, corrobora os achados de diversos autores, que enfatizam a grande diferença das prevalências encontradas entre os sexos, além de salientar o problema socioeconômico decorrente destes, tais como o comprometimento não somente da vítima envolvida, mas de toda a família e comunidade à qual pertence, além dos custos para o Estado no caso das seqüelas definitivas e incapacidade da vítima em retornar à atividade laborativa ${ }^{4,7,9,10,12,13}$.

Os acidentes automobilísticos destacam-se como principais causas de traumas mecânicos ${ }^{1,6,12}$. Sala e cols..$^{14}$ constataram que a principal causa de traumas múltiplos é o acidente com meios de transporte, o que corrobora os resultados do presente estudo, sendo o atropelamento o principal responsável. 
Elevadas taxas de traumas múltiplos em vítimas de TCE são descritas por outros autores em diferentes mecanismos de trauma ${ }^{5,13}$. Em relação aos principais locais acometidos concomitantes ao TCE, este trabalho pode ser ratificado pelos resultados encontrados por Sala e cols..$^{14}$ e Melo e cols. ${ }^{13}$, que evidenciaram maior prevalência do trauma ósseo nos membros e na face em associação com o trauma em crânio.

Constatando o predomínio do TCE grave em vítimas de traumas múltiplos, verifica-se a importância da investigação diagnóstica exaustiva nesses pacientes, em busca de lesões associadas, para que se possam oferecer, sempre, as melhores condutas terapêuticas, tendo em vista a dificuldade do exame clínico acurado em virtude do importante rebaixamento do nível de consciência ${ }^{5}$.

\section{Conclusão}

Por meio da análise dos dados, conclui-se que a associação entre trauma de crânio e o de outra região do corpo ocorreu em $31,9 \%$ dos casos, sendo a face o principal local de trauma concomitante ao TCE, seguido do trauma ortopédico nos membros, tendo como principal causa o atropelamento.

Verificou-se, também, que as principais vítimas de traumas múltiplos foram os adultos jovens do sexo masculino, admitidos com diagnóstico de TCE grave.

\section{Agradecimentos}

Aos funcionários do Serviço de Arquivo Médico do HGE, pela disponibilidade e pelo auxílio durante a pesquisa nos prontuários médicos.

\section{Referências}

1. ANDRADE AF, MANREZA LA, GIUDICISSI Filho $M$, MIURA FK: Normas de Atendimento ao paciente com traumatismo crânio-encefálico. Temas Atuais em Neurocirurgia. São Paulo, SONESP, 1996.
2. ANDRADE AF, FIGUEIREDO EG, BROCK RS: Orientação aos familiares e pacientes que sofreram traumatismo craniano-cerebral. Disponivel em: <http:// wnw.sbn.com.br/programas/pensebem.htm>Acesso em 30 de março de 2003.

3. BORDIGNON KC, ARRUDA WO: CT scan findings in mild head trauma. A series of 2000 patients. Arq Neuropsiquiatr 60:204-10, 2002.

4. BOSWELL JE, McERLEAN M, VERDILE VP: Prevalence of traumatic brain injury in an ED population. Am J Emerg Med 20:177-80, 2002.

5. BUDUHAN G, MCRITCHIE DI: Missed injuries in patients with multiple trauma. J Trauma 49:600-5, 2000.

6. FARAGE L, COLARES VS, CAPP NETO M, MORAES MC, BARBOSA MC, BRANCO JÚNIOR JA: As medidas de segurança no trânsito e a morbimortalidade intrahospitalar por traumatismo craniencefálico no Distrito Federal. Rev Assoc Med Bras 48:133-6, 2002.

7. FINFER SR, COHEN J: Severe traumatic brain injury. Resuscitation 48:77-90, 2001.

8. KAY A, TEASDALE G: Head injury in the United Kingdom. World J Surg 25:1210-20, 2001.

9. KOIZUME MS, LEBRÃO ML, MELLO-JORGE MHP, PRIMERANO V: Morbimortalidade por traumatismo crânio-encefálico no município de São Paulo, 1997. Arq Neuropsiquiatr 58:1-13, 2000.

10. KRAUSS JF, MCARTHUR DL: Epidemiology of brain injury. In Evans RW (ed): Neurology and trauma. Ed 1. HoustonTexas, W.B. Saunders, 1996, cap 1, pp 3-17.

11. MACKENZIE EJ: Epidemiology of injuries: Current trends and future challenges. Epidemiol Rev 22:112-9, 2000.

12. MASINI M: Perfil epidemiológico do traumatizado craniencefálico no Distrito Federal 1991. Dissertação de Mestrado. Escola Paulista de Medicina, Universidade Federal de São Paulo. São Paulo, 1994.

13. MELO JRT, SILVA RA, SILVA LGA, HERMIDA MB: Características do trauma craniofacial no Hospital Geral do Estado da Bahia. Ciência e Saúde 31:31-5, 2003.

14. SALA D, FERNANDEZ E, MORANT A, GASCO J, BARRIOS C: Epidemiologic aspects of pediatric multiple trauma in a Spanish urban population. J Pediatr Surg 3510:1478-81, 2000.

15. ZAFONTE RS, WOOD DL, HARRISON-FELIX CL, MILLIS SR, VALENA NV: Severe penetrating head injury: a study of outcomes. Arch Phys Med Rehabil 82:30610, 2001.

Original recebido em janeiro de 2004

Aceito para publicação em abril de 2004

\section{Endereço para correspondência:}

José Roberto Tude Melo

Hospital São Rafael

Avenida São Rafael, $2152-3^{\circ}$ andar - setor $A$

CEP 41256-900-Salvador, BA

E-mail: robertotude@hotmail.com 